\title{
Geometrical equivalence and action type geometrical equivalence of group representations*
}

\author{
J. Simões da Silva and A. Tsurkov
}

\author{
Communicated by I. P. Shestakov
}

\begin{abstract}
A BSTRACT. In this paper we construct an example of two representations $\left(V_{1}, G_{1}\right)$ and $\left(V_{2}, G_{2}\right)$ which are action type geometrically equivalent and groups $G_{1}$ and $G_{2}$ are geometrically equivalent, but the representations $\left(V_{1}, G_{1}\right)$ and $\left(V_{2}, G_{2}\right)$ are not geometrically equivalent.
\end{abstract}

\section{Introduction}

All definitions of the basic notions of the universal algebraic geometry can be found, for example, in [5], [6], [7] and [8]. Also, there are fundamental papers [1], [4] and [2], [3].

Some problems of the universal algebraic geometry for many-sorted universal algebras were considered also in [10], [12], [13] and [14].

Let $K$ be an arbitrary but fixed field. We consider representations of groups over $K$-vector spaces. In this paper a representation of group is a pair $(V, G)$, where $V$ is a vector space over field $K$ and $G$ is a group. The signature of this algebraic object includes all operations of the vector space $V$ (multiplication by a scalar $\lambda \in K$ we consider as unary operation: $\lambda: V \rightarrow V$, where $\lambda(v)=\lambda v, v \in V)$, all operations of the group $G$ and

${ }^{*}$ The authors acknowledge the support of Coordenação de Aperfeiçoamento de Pessoal de Nível Superior - CAPES (Coordination for the Improvement of Higher Education Personnel, Brazil).

2010 MSC: 20C99, 08C10.

Key words and phrases: universal algebraic geometry, group representations. 
the operation of action of $G$ on vector space $V$. We denote this operation by $\circ$ :

$$
V \times G \ni(v, g) \rightarrow v \circ g \in V .
$$

From now on we will write briefly "representation" instead of "representation of group". The homomorphism $(\alpha, \beta):(V, G) \rightarrow(W, H)$ from representation $(V, G)$ to representation $(W, H)$ is a pair, where $\alpha: V \rightarrow W$ is a linear mapping and $\beta: G \rightarrow H$ is a homomorphism of groups, such that for every $v \in V$ and every $g \in G$ the equality

$$
\alpha(v \circ g)=\alpha(v) \circ \beta(g)
$$

holds. The reader can see that we consider representations as 2-sorted universal algebras: the first sort is a sort of vectors from a vector space and the second sort is a sort of elements from a group. This approach to representations can be found in [11], [15], [10], [13].

The variety of all representations of groups over a fixed field $K$ will be denoted by REP- $K$.

Definition 0.1. The representation $W(X, Y)=(U(X, Y), H(X, Y))$ is called the free representation generated by sets $X$ and $Y$ if, for every $(V, G) \in$ REP- $K$ and every mappings $f_{1}: X \rightarrow V$ and $f_{2}: Y \rightarrow G$, there is a unique homomorphism of representations $(\alpha, \beta):(U(X, Y), H(X, Y)) \rightarrow$ $(V, G)$, such that $\alpha_{\mid X}=f_{1}, \beta_{\mid Y}=f_{2}$.

It was proved in [11], that $(U(X, Y), H(X, Y))=(X K F(Y), F(Y))$, where $F(Y)$ is the free group with the free set of generators $Y, K F(Y)$ is the group ring over $F(Y)$ and $X K F(Y)=\bigoplus_{x \in X} x K F(Y)$ is the free $K F(Y)$-module with free basis $X$.

\section{Basic notions of the algebraic geometry of representations}

From now on we suppose that sets $X$ and $Y$ of generators of the free representations $(X K F(Y), F(Y))$ are finite. Equations in the algebraic geometry of representations have the form $v_{1}=v_{2}$, where $v_{1}, v_{2} \in X K F(Y)$, or the form $f_{1}=f_{2}$ where $f_{1}, f_{2} \in F(Y)$ The equation of the first form is equivalent to the equation $v_{1}-v_{2}=0$, and the equation of the second form is equivalent to the equation $f_{1} f_{2}^{-1}=1$. So, in algebraic geometry of representations we can consider the system of equations $T=\left(T_{1}, T_{2}\right)$, where $T_{1} \subseteq X K F(Y), T_{2} \subseteq F(Y)$, or, briefly, $\left(T_{1}, T_{2}\right) \subseteq(X K F(Y), F(Y))$. If we look for solutions of this system of equations in the representation 
$(V, G) \in \mathrm{REP}-K$, then the set $\operatorname{Hom}((X K F(Y), F(Y)),(V, G))$ takes the role of the affine space. The solution of the system $\left(T_{1}, T_{2}\right)$ in $(V, G)$ is the set

$$
\begin{aligned}
\left(T_{1}, T_{2}\right)_{(V, G)}^{\prime}=\left\{(\alpha, \beta) \in \operatorname{Hom}((X K F(Y), F(Y)),(V, G)) \mid T_{1} \subseteq \operatorname{ker}(\alpha)\right. \\
\left.\quad \text { and } T_{2} \subseteq \operatorname{ker}(\beta)\right\}
\end{aligned}
$$

The algebraic $(V, G)$-closure of the system $\left(T_{1}, T_{2}\right)$ is defined as

$$
\begin{aligned}
& \left(T_{1}, T_{2}\right)_{(V, G)}^{\prime \prime} \\
& \quad=\left(\bigcap_{(\alpha, \beta) \in\left(T_{1}, T_{2}\right)_{(V, G)}^{\prime}} \operatorname{ker}(\alpha), \bigcap_{(\alpha, \beta) \in\left(T_{1}, T_{2}\right)_{(V, G)}^{\prime}} \operatorname{ker}(\beta)\right) \subseteq(X K F(Y), F(Y)) .
\end{aligned}
$$

This is the maximal system of equations, which has the same solutions as the system $\left(T_{1}, T_{2}\right)$. It is easy to see that the transition ' defines a kind of the Galois correspondence (see [10] for details).

Definition 1.1. Let $\left(V_{1}, G_{1}\right),\left(V_{2}, G_{2}\right) \in \operatorname{REP}-K$. We say that $\left(V_{1}, G_{1}\right)$ and $\left(V_{2}, G_{2}\right)$ are geometrically equivalent if $\left(T_{1}, T_{2}\right)_{\left(V_{1}, G_{1}\right)}^{\prime \prime}=\left(T_{1}, T_{2}\right)_{\left(V_{2}, G_{2}\right)}^{\prime \prime}$, for every $\left(T_{1}, T_{2}\right) \subseteq(X K F(Y), F(Y))$ and every $X$ and $Y$. We use the notation $\left(V_{1}, G_{1}\right) \sim\left(V_{2}, G_{2}\right)$.

The notion of geometric equivalence can be defined in an arbitrary variety of universal algebras. For more details, see [5].

Here we introduce the notion of identity of a representation.

Consider logical formulas of the form

$$
v=0
$$

where $v \in X K F(Y)$, or

$$
f=1
$$

where $f \in F(Y)$.

Definition 1.2. Let $(V, G) \in \operatorname{REP}-K$. We say that $(V, G)$ satisfies the identity (1.1) if, for every $(\alpha, \beta) \in \operatorname{Hom}((X K F(Y), F(Y)),(V, G))$ the equality $\alpha(v)=0$ holds. We say that $(V, G)$ satisfies the identity (1.2) if, for every $(\alpha, \beta) \in \operatorname{Hom}((X K F(Y), F(Y)),(V, G))$ the equality $\beta(f)=1$ holds. 
We will write

$$
(V, G) \vDash(v=0)
$$

in the first case and

$$
(V, G) \vDash(f=1)
$$

in the second case.

For example the identity $y \circ(x-1)^{n}=0$ defines the variety of all unipotent representations of degree $n$.

Definition 1.3. The logic formula of the form

$$
\left(\bigwedge_{i=1}^{n} w_{i}\right) \Rightarrow w_{0}
$$

where $w_{i}$ can have either form of (1.1) or form of (1.2), $0 \leqslant i \leqslant n, n \in \mathbb{N}$, is called quasi-identity.

Definition 1.4. Let $(V, G) \in \operatorname{REP}-K$. We say that $(V, G)$ satisfies the quasi-identity (1.3) if, for every $(\alpha, \beta) \in \operatorname{Hom}((X K F(Y), F(Y)),(V, G))$ which satisfies the conditions: for $1 \leqslant i \leqslant n$,

$1)$ if $w_{i}$ is $\left(v_{i}=0\right)$ then $\alpha\left(v_{i}\right)=0$,

$2)$ if $w_{i}$ is $\left(f_{i}=1\right)$ then $\beta\left(f_{i}\right)=1$, we have that

- if $w_{0}$ is $\left(v_{0}=0\right)$ then $\alpha\left(v_{0}\right)=0$, or

- if $w_{0}$ is $\left(f_{0}=1\right)$ then $\beta\left(f_{0}\right)=1$.

We will write

$$
(V, G) \vDash\left(\left(\bigwedge_{i=1}^{n} w_{i}\right) \Rightarrow w_{0}\right),
$$

if $(V, G)$ satisfies the quasi-identity (1.3).

By [9, Theorem 2] we have

Proposition 1.1. Let $\left(V_{1}, G_{1}\right),\left(V_{2}, G_{2}\right) \in \operatorname{REP}-K$ and $\left(V_{1}, G_{1}\right) \sim$ $\left(V_{2}, G_{2}\right)$ then $\left(V_{1}, G_{1}\right)$ and $\left(V_{2}, G_{2}\right)$ satisfy same quasi-identities.

By [5, Proposition 13] we also have the following.

Proposition 1.2. Let $\Theta$ be some variety of universal algebras and $H_{1}, H_{2} \in \Theta$. Then $H_{1} \sim H_{2}$ if and only if for every finitely generated subalgebra $H_{1}^{0} \subset H_{1}$ there exists an injection $H_{1}^{0} \hookrightarrow H_{2}^{I_{2}}$ and for every finitely generated subalgebra $H_{2}^{0} \subset H_{2}$ there exists an injection $H_{2}^{0} \hookrightarrow H_{1}^{I_{1}}$, where $I_{1}, I_{2}$ are some sets of indexes and $H_{1}^{I_{1}}, H_{2}^{I_{2}}$ are the corresponding Cartesian powers of algebras $H_{1}$ and $H_{2}$. 
Corollary 1. If $H_{1}, H_{2}$ are finitely generated algebras from $\Theta$ then $H_{1} \sim H_{2}$ if and only if there exist injections $H_{1} \hookrightarrow H_{2}^{I_{2}}$ and $H_{2} \hookrightarrow H_{1}^{I_{1}}$, where $I_{1}, I_{2}$ are some sets of indexes and $H_{1}^{I_{1}}, H_{2}^{I_{2}}$ corresponding Cartesian powers of algebras $H_{1}$ and $H_{2}$.

In [10] the action type algebraic geometry of representations was considered. This geometry was studied in order to avoid the influence of the algebraic geometry of the acting group on the algebraic geometry of representation.

In this geometry we consider only system of action type equations, i.e., system of equations which have form $T \subseteq X K F(Y)$. The set of solutions of this system in the representation $(V, G)$ is the set

$$
T_{(V, G)}^{\prime}=\{(\alpha, \beta) \in \operatorname{Hom}((X K F(Y), F(Y)),(V, G)) \mid T \subseteq \operatorname{ker}(\alpha)\} .
$$

The action type $(V, G)$-closure of the system of equations $T$ is a set

$$
T_{(V, G)}^{\prime \nabla}=\bigcap_{(\alpha, \beta) \in T_{(V, G)}^{\prime}} \operatorname{ker}(\alpha) \subseteq X K F(Y) .
$$

This is the maximal system of action type equations, which has the same solutions as the system $T$.

Definition 1.5. Let $\left(V_{1}, G_{1}\right),\left(V_{2}, G_{2}\right) \in \operatorname{REP}-K$. We say that $\left(V_{1}, G_{1}\right)$ and $\left(V_{2}, G_{2}\right)$ are action type geometrically equivalent if $T_{\left(V_{1}, G_{1}\right)}^{\prime \nabla}=T_{\left(V_{2}, G_{2}\right)}^{\prime \nabla}$, for every $T \subseteq X K F(Y)$ and every $X$ and $Y$. We use the notation $\left(V_{1}, G_{1}\right) \sim_{a t}\left(V_{2}, G_{2}\right)$.

By [10, Corollary 2 from Proposition 4.2], if two representations $\left(V_{1}, G_{1}\right)$ and $\left(V_{2}, G_{2}\right)$ are geometrically equivalent then they are action type geometrically equivalent.

Example 1. We consider the representation $(V, G)$. Also we can consider the vector space $V_{1}=V^{I}$, which is a Cartesian power of $V$ indexed by the set $I$; and the group $G_{1}=G^{I}$, which is a Cartesian power of $G$ indexed by the same set. We can define the action of $G_{1}$ in $V_{1}$ component-wise, i.e.: for every $v=\left(v_{i}\right)_{i \in I} \in V_{1}$ and every $g=\left(g_{i}\right)_{i \in I} \in G_{1}$ we define $(v \circ g)_{i}=v_{i} \circ g_{i}$ for each coordinate $i$. It is clear that this action gives us the representation $\left(V_{1}, G_{1}\right)$. By Proposition 1.2 the representations $(V, G)$ and $\left(V_{1}, G_{1}\right)$ are geometrically equivalent. Therefore these representations are action type geometrically equivalent. The groups $G$ and $G_{1}$ are also geometrically equivalent. 
Example 2. We can prove by method of [9, Proposition 4] that the natural representations of the groups $G_{1}=\mathbf{U T}_{n}(\mathbb{Z})$ and $G_{2}=\mathbf{U T}_{n}(\mathbb{Q})$ in $V=\mathbb{Q}^{n}$ are geometrically equivalent. In this example, we have the same situation as in the previous one. The representations $\left(V, G_{1}\right)$ and $\left(V, G_{2}\right)$ are geometrically equivalent. Therefore these representations are action type geometrically equivalent. Here also the groups $G_{1}$ and $G_{2}$ are geometrically equivalent.

More examples of the action type geometric equivalence of representations can be obtained from Proposition 1.3.

If $(V, G) \in \mathrm{REP}-K$, for every $v \in V$ we can consider the stabilizer of $v$, defined as follows

$$
\operatorname{stab}(v)=\{g \in G \mid v \circ g=v\},
$$

and $\operatorname{ker}(V, G)$, defined as follows

$$
\operatorname{ker}(V, G)=\bigcap_{v \in V} \operatorname{stab}(v) .
$$

One can prove that $\operatorname{ker}(V, G)$ is a normal subgroup of $G$. We denote by $\tilde{G}$ the quotient group $G / \operatorname{ker}(V, G)$ and by $\sigma$ the natural epimorphism $\sigma$ : $G \rightarrow G / \operatorname{ker}(V, G)$. It also is easy to check that we obtain the representation $(V, \tilde{G})$ if we define the action of the group $\tilde{G}$ on $V$ as follows

$$
v \circ \sigma(g)=v \circ g,
$$

where $v \in V, g \in G$.

Definition 1.6. The representation $(V, \tilde{G})$ is called the faithful image of the representation $(V, G)$.

By [10, Corollary 4 from Theorem 5.1], we have

Proposition 1.3. Every representation $(V, G) \in \mathrm{REP}-K$ is action type geometrically equivalent to its faithful image $(V, \tilde{G})$.

\section{The relation between geometrical equivalence and action type geometrical equivalence of group representations}

In this section we will discuss the following question: 
Problem 1. Let $\left(V_{1}, G_{1}\right),\left(V_{2}, G_{2}\right) \in$ REP- $K$. Does the geometric equivalence $\left(V_{1}, G_{1}\right) \sim\left(V_{2}, G_{2}\right)$ follow from the action type geometric equivalence $\left(V_{1}, G_{1}\right) \sim_{a t}\left(V_{2}, G_{2}\right)$ and the geometrical equivalence of groups $G_{1} \sim G_{2}$ ?

The negative answer to this question was mentioned in [10, Remark 5.1], but no counterexample was presented. This question makes sense, because in the action type algebraic geometry of representations we consider only specific systems of equations which have form $T \subseteq X K F(Y)$ and the specific form of the algebraic closure (1.4). By this restriction we avoid the influence of the algebraic geometry of acting groups of representations. The question that comes naturally is whether we are losing some important information about representations by this restriction. The negative answer to the Problem 1 shows that we indeed lose some information.

Theorem 2.1. There exists $\left(V_{1}, G_{1}\right),\left(V_{2}, G_{2}\right) \in$ REP- $K$, such that $\left(V_{1}, G_{1}\right) \sim_{a t}\left(V_{2}, G_{2}\right), G_{1} \sim G_{2}$, but $\left(V_{1}, G_{1}\right) \nsim\left(V_{2}, G_{2}\right)$.

Proof. We consider a vector space $V$ over an arbitrary field $K$, such that $\operatorname{dim}_{K}(V)=2$. Let $\left\{e_{1}, e_{2}\right\}$ be a basis of $V$. Also we consider the groups $G_{1}=\langle a\rangle \cong \mathbb{Z}_{2}$ and $G_{2}=\langle a\rangle \times\langle b\rangle \cong \mathbb{Z}_{2} \times \mathbb{Z}_{2}$. In order to define the representation of a group $G$ on a vector space $V$ it is enough to define a homomorphism from this group to the group $\mathbf{G L}(V)$ of all invertible linear operators on vector space $V$. We define the homomorphism $\varphi$ of $G_{2}$ to the group $\langle S\rangle<\mathbf{G} \mathbf{L}(V)$, where $S$ is the invertible linear operator on vector space $V$ such that $\left(e_{1}\right) S=e_{2},\left(e_{2}\right) S=e_{1}$. Let us set: $\varphi(a)=S$, $\varphi(b)=i d_{V} \cdot G_{2}=\langle a\rangle \times\langle b\rangle$, so this homomorphism is defined correctly. $G_{1}$ is a subgroup of $G_{2}$, so we also have a homomorphism $\varphi_{\mid G_{1}}: G_{1} \rightarrow\langle S\rangle$. We can say that generators of the groups $G_{1}$ and $G_{2}$ act on elements of basis of $V$ in this way:

$$
\begin{aligned}
& e_{1} \circ a=e_{2}, \quad e_{2} \circ a=e_{1}, \\
& e_{1} \circ b=e_{1}, \quad e_{2} \circ b=e_{2} .
\end{aligned}
$$

Hence, we obtain the representations $\left(V_{1}, G_{1}\right)$ and $\left(V_{2}, G_{2}\right)$, where $V_{1}=V_{2}=V$. Note that $\operatorname{ker}\left(V, G_{2}\right)=\langle b\rangle \cong \mathbb{Z}_{2}$ and $G_{2} / \operatorname{ker}\left(V, G_{2}\right) \cong$ $G_{1}=\langle a\rangle \cong \mathbb{Z}_{2}$. Therefore, the faithful image $\left(V, \widetilde{G_{2}}\right)$ of the representation $\left(V, G_{2}\right)$ is isomorphic to the representation $\left(V, G_{1}\right)$. So, by Proposition 1.3, $\left(V, G_{1}\right) \sim_{a t}\left(V, G_{2}\right)$.

Obviously, there are injections $G_{1} \hookrightarrow G_{2}$ and $G_{2} \hookrightarrow G_{1} \times G_{1}$. Therefore, by Corollary 1 from Proposition 1.2, $G_{1} \sim G_{2}$. 
Now, we consider the quasi-identity

$$
(x \circ y-x=0) \Rightarrow(y=1) .
$$

We have that

$$
\left(V, G_{1}\right) \vDash((x \circ y-x=0) \Rightarrow(y=1)),
$$

because $\operatorname{ker}\left(V, G_{1}\right)=\{1\}$, and

$$
\left(V, G_{2}\right) \not \models((x \circ y-x=0) \Rightarrow(y=1)),
$$

because $\operatorname{ker}\left(V, G_{2}\right) \neq\{1\}$. By Proposition 1.1 this means that $\left(V_{1}, G_{1}\right) \nsim$ $\left(V_{2}, G_{2}\right)$.

\section{Acknowledgement}

We are thankful to Professor E. Aladova for her important remarks, which helped a lot in writing this article.

\section{References}

[1] G. Baumslag, A. Myasnikov, V. Remeslennikov, Algebraic geometry over groups I: Algebraic sets and ideal theory, Journal of Algebra, 219, 1999, pp.16-79.

[2] E. Daniyarova, A. Myasnikov, V. Remeslennikov, Algebraic geometry over algebraic structures II: Foundations, J. Math. Sci., 185:3, 2012, pp.389-416.

[3] E. Daniyarova, A. Myasnikov, V. Remeslennikov, Algebraic geometry over algebraic structures $V$. The case of arbitrary signature, Algebra Logic, 51:1, 2012, pp.28-40.

[4] A. Myasnikov, V. Remeslennikov, Algebraic geometry over groups II: Logical Foundations, Journal of Algebra, 234, 2000, pp.225-276.

[5] B. Plotkin, Varieties of algebras and algebraic varieties. Categories of algebraic varieties, Siberian Advanced Mathematics, Allerton Press, 7:2, 1997, pp.64-97.

[6] B. Plotkin, Some notions of algebraic geometry in universal algebra, Algebra and Analysis, 9:4, 1997, pp.224-248, St. Petersburg Math. J., 9:4, 1998, pp.859-879.

[7] B. Plotkin, Algebras with the same (algebraic) geometry, Proceedings of the International Conference on Mathematical Logic, Algebra and Set Theory, dedicated to 100 anniversary of P.S. Novikov, Proceedings of the Steklov Institute of Mathematics, MIAN, 242, 2003, pp.17-207.

[8] B. Plotkin, E. Plotkin, Multi-sorted logic and logical geometry: some problems, Demonstratio Mathematica, XLVIII (4), 2015, pp.578-618.

[9] B. Plotkin, E. Plotkin, A. Tsurkov. Geometrical equivalence of groups, Communications in Algebra, 27(8), 1999, pp.4015-4025.

[10] B. Plotkin, A. Tsurkov. Action type geometrical equivalence of group representations, Algebra and discrete mathematics, 4, 2005, pp.48-79. 
[11] B. Plotkin, S. Vovsi. Varieties of representations of groups, Zinatne, Riga, 1983 (in Russian).

[12] I. Shestakov, A. Tsurkov, Automorphic equivalence of the representations of Lie algebras, Algebra and Discrete Mathematics, 15, 2013, pp.96-126.

[13] A. Tsurkov, Automorphic equivalence of many-sorted algebras, Applied Categorical Structures, 24:3, 2016, pp.209-240.

[14] A. Tsurkov, Automorphic Equivalence in the Varieties of Representations of Lie algebras, Communications in Algebra, 48:1, 2020, pp.397-409.

[15] S. M. Vovsi, Topics in varieties of group representations, Cambridge University Press, 1991.

\section{CONTACT INFORMATION}

Josenildo Simões Mathematical Department, CCET, da Silva, Federal University of Rio Grande do Norte, Arkady Tsurkov Av. Senador Salgado Filho, 3000, Campus Universitário, Lagoa Nova, Natal - RN - Brazil - CEP 59078-970

E-Mail(s): josenildosimoesdasilva@gmail.com, arkady .tsurkov@gmail.com

Received by the editors: 20.04.2018

and in final form 25.06.2020. 